\title{
Correction to: Her2 promotes early dissemination of breast cancer by suppressing the p38 pathway through Skp2-mediated proteasomal degradation of $\mathrm{Tpl} 2$
}

Guanwen Wang · Juan Wang · Antao Chang - Dongmei Cheng · Shan Huang - Dan Wu - Sherona Sirkisoon • Shuang Yang $\cdot$ Hui-Kuan Lin (D) $\cdot$ Hui-Wen Lo (D) Rong Xiang (D) Peiqing Sun (D)

Published online: 10 February 2021

(C) The Author(s), under exclusive licence to Springer Nature Limited 2021

Correction to: Oncogene

https://doi.org/10.1038/s41388-020-01481-y
In the original published version Institution \#1 and \#2 were switched. The original article was corrected. 